\title{
CONSTRUCTION BY ISOTOPY. II
}

\author{
DANIEL S. SILVER
}

\begin{abstract}
Construction by isotopy is a technique introduced by Iain R. Aitchison for obtaining doubly slice fibered knots in any dimension. We show that if $k$ is any doubly slice fibered $(n-2)$-knot, $n \geq 5$, such that $\pi_{1}\left(S^{n}-k\right) \cong Z$, then $k$ is constructible by isotopy. We also prove that the $m$-twist-spin of any doubly slice knot is constructible by isotopy. Consequently, there exists a double slice knot constructible by isotopy that is not the double of any disk knot. We also give an example of a doubly slice fibered 6-knot that is not constructible by isotopy.
\end{abstract}

We continue an investigation [Sil] of construction by isotopy, a technique introduced by Iain R. Aitchison [A] for obtaining doubly slice fibered knots of any dimension. We introduce the problem of finding a fibered double slice for a knot, and show that in higher dimensions this problem is equivalent to producing the knot by the construction. As a result, we are able to expand the class of doubly slice fibered knots that are known to be constructible by isotopy. We show, for example, that any twist-spin of a doubly slice knot (of any dimension) is constructible by isotopy. Using a result of Levine [Le] we then confirm that construction by isotopy can produce knots that are not doubles of disk knots; this provides an affirmative answer to a question posed in [A, p. 67]. On the other hand, work of Cappell and Ruberman [CaR, $R_{1}, R_{2}$ ] enables us to give an example of a doubly slice fibered 6-knot that does not arise from the construction; consequently, we disprove Aitchison's main conjecture [A, Sil]: Every doubly slice fibered knot is constructible by isotopy.

All manifolds that appear are smooth and oriented. We will smooth corners when they appear. Maps are smooth. The symbols cl, int, nbd denote closure, interior and tubular neighborhood, respectively. Homology groups have integer coefficients and homomorphisms are induced by inclusion unless otherwise indicated.

An $(n-2)$-knot, $n \geq 3$, is a submanifold $k$ of the $n$-sphere $S^{n}$ diffeomorphic to $S^{n-2}$. The knot is trivial if it bounds an embedded ball. The knot $k$ is fibered if $X=\operatorname{cl}\left(S^{n}-\operatorname{nbd}(k)\right)$ admits a locally trivial fibration $f: X \rightarrow S^{1}$, trivial on the boundary $\partial X$. (We will refer to $f$ as a fibration of $k$.) In this case, we can identify $X$ with a mapping torus $F \times{ }_{h} S^{1}=F \times I /\{(x, 0) \sim(h(x), 1)\}$,

Received by the editors June 24, 1988.

1980 Mathematics Subject Classification (1985 Revision). Primary 57M25, 57Q45.

Partially supported by the University of South Alabama Research Council. 
where $F$ is an $(n-1)$-manifold with boundary $k$ and $h: F \rightarrow F$ is a diffeomorphism. We will call $h$ the characteristic map and $F$ the fiber of the fibration.

An $(n-1)$-disk knot, $n \geq 2$, is a properly embedded $(n-1)$-ball $D^{n-1}$ in $B^{n+1}$. If $n>2$, the $(n-2)-k n o t ~ \partial D^{n-1} \subset \partial B^{n+1}=S^{n}$ is said to be slice and $D^{n-1}$ is called a slice disk for the knot. A disk knot $D^{n-1}$ is fibered if $Y=\operatorname{cl}\left(B^{n+1}-\operatorname{nbd}\left(D^{n-1}\right)\right)$ admits a locally trivial fibration $g: Y \rightarrow S^{1}$, trivial on $D^{n-1} \times \partial D^{2} \subset \partial Y$. (We will refer to $g$ as a fibration of $D^{n-1}$. Notice that $\partial D^{n-1}$ is a fibered $(n-2)$-knot.) The terms characteristic map and fiber are defined as for fibered knots. A disk knot $D_{1}^{n-1}$ is invertible if there exists another disk knot $D_{2}^{n-1}$, called an inverse (disk knot) for $D_{1}^{n-1}$, such that $\partial D_{1}^{n-1}=\partial D_{2}^{n-1}$ and $D_{1}^{n-1} \cup_{\partial} D_{2}^{n-1}$ is a trivial $(n-1)$-knot in $B^{n+1} \cup_{\partial} B^{n+1}=S^{n+1}$. In this case, the $(n-2)$-knot $k=\partial D^{n-1}$ is said to be doubly slice, provided $n>2$, and we will call the pair $D_{1}^{n-1}, D_{2}^{n-1}$ a double slice for $k$. Finally, a doubled disk knot is the $(n-1)$-knot formed by the union $D^{n-1} \cup_{\partial} D^{n-1} \subset B^{n+1} \cup_{\partial} B^{n+1}=S^{n+1}$, where $D^{n-1} \subset B^{n+1}$ is any disk knot.

An $(n-2)$-knot $k$ is constructible by isotopy if there exists a compact connected $n$-dimensional submanifold $\Xi \subset S^{n}$ and an isotopy $h_{t}: S^{n} \rightarrow S^{n}$, $0 \leq t \leq 1$, with the following properties:

(1) $h_{0}$ is the identity map 1 , while $h_{1}$ restricts to a diffeomorphism $\phi: \Xi \rightarrow$ $\Xi$.

(2) $\partial \Xi$ contains a small $(n-1)$-ball $\mathbb{D}$ such that $h_{t}$ preserves $\mathbb{D}$ set-wise with $h_{1}$ restricting to the identity map.

(3) $\Xi X_{\phi} S^{1} \cup\left(2\right.$-handle attached along $\left.\mathrm{D} \times S^{1}\right)=B^{n+1}$ with $\partial \mathbb{D} \times \overline{0}=k$. Aitchison has shown [A] that any knot $k$ that arises in this manner is doubly slice and fibered-in fact, $\mathbf{D}(=\mathbf{D} \times \overline{0}) \subset B^{n+1}$ is an invertible fibered slice disk for $k$. (See [Sil] also.)

I wish to express my deepest appreciation to Cherry Kearton for his helpful suggestions and encouragement, and I am grateful to the University of Durham for its hospitality. I also wish to thank Daniel Ruberman for several helpful discussions.

\section{DOUBLES OF FIBERED DISK KNOTS}

In [A] Aitchison proved that the connected-sum $k \#-k$ is constructible by isotopy for any fibered $(n-2)-k n o t k, n \geq 3$. Notice that $k \#-k$ is the double of the fibered disk knot that we obtain by removing a standard ball pair from $\left(S^{n}, k\right)$. Aitchison's result extends:

Theorem 1.1. The double of any fibered disk knot is constructible by isotopy.

Proof. Our proof closely follows Aitchison's argument [A, pp. 61-62] that $k \#$ $-k$ is constructible by isotopy for any fibered knot $k$. 
Let $F$ and $h$ denote the fiber and characteristic map, respectively, for a fibration of any disk knot $D^{n-2} \subset B^{n}$. Consider the product disk knot $D^{n-2} \times$ $I \subset B^{n} \times I$, which is also fibered, with fiber $F \times I$ and characteristic map $h \times 1$. Henceforth, we will identify $\partial\left(D^{n-2} \times I\right)$ with the double of $D^{n-2}$.

Embed $\operatorname{cl}\left(B^{n} \times I-\operatorname{nbd}\left(D^{n-2} \times I\right)\right)$ into $S^{n} \times S^{1}$ as follows: First embed $\operatorname{cl}\left(B^{n}-\operatorname{nbd}\left(D^{n-2}\right)\right)$, regarded as $F \times{ }_{h} S^{1}$, fiberwise in $S^{n} \times S^{1}$. (The covering homotopy theorem provides a one-parameter family of embeddings $\eta_{\theta}: F \rightarrow$ $g^{-1}(\bar{\theta}) \subset B^{n} \subset S^{n}$, where $g$ is a fibration of $D^{n-2}$; then the map $x \times \bar{\theta} \rightarrow$ $\eta_{\theta}(x) \times \bar{\theta}, \theta \in I$, is the desired embedding.) Next, thicken each copy of $F$ in its factor $S^{n} \times \bar{\theta}$. Note that the normal bundle of $\operatorname{cl}\left(B^{n}-\operatorname{nbd}\left(D^{n-2}\right)\right) \subset S^{n} \times S^{1}$ is a one-dimensional orientable bundle and hence is trivial; consequently, there is no difficulty thickening all the copies of $F$ at once.

Identify the disk knot $D^{n-2}$ with $D^{n-2} \times \overline{0}$ in the boundary of $\operatorname{cl}\left(B^{n}-\operatorname{nbd}\left(D^{n-2}\right)\right)$. Since the characteristic map $h$ fixes $D^{n-2}$ pointwise, the map $h \times 1$ fixes $D^{n-2} \times I \subset \partial(F \times I)$ pointwise. Denote $F \times I$ and $D^{n-2} \times I$ by $\Xi$ and $\mathbb{D}$, respectively. With the help of the embedding of $\operatorname{cl}\left(B^{n} \times I-\operatorname{nbd}\left(D^{n-2} \times I\right)\right)$ into $S^{n} \times S^{1}$ described above, we can regard $h \times 1$ as the restriction to $\Xi$ of an isotopy (relD) of $S^{n}$. By attaching a 2-handle to the mapping torus $\Xi \times_{h \times 1} S^{1}$ along $\mathrm{D} \times S^{1}$, we recover $B^{n} \times I$ containing $\partial\left(D^{n-2} \times I\right) \quad(=\partial \mathbf{D} \times \overline{0})$. Hence the double of $D^{n-2}$ is constructible by isotopy.

Remark. In the classical dimension $(n=3)$ it is possible to introduce additional deformations of $\Xi$ during the isotopy of $S^{3}$ and thereby produce infinitely many distinct doubly slice fibered 1-knots with identical Alexander modules. This was originally done in $[\mathrm{A}]$ where the resulting knots, derived from $4 \# 4$, were distinguished using stretching factors of their pseudo-Anosov characteristic maps. Other examples, distinguished using the Jones polynomial, were produced in [HiSil].

\section{Fibered DOUbLE SLICES}

In this section we will show that if any doubly slice fibered knot is constructible by isotopy, then there exists a fibration of the knot that extends to each disk knot of some double slice for the knot. The converse is true, except possibly in the two lowest dimensions, and will provide a useful way to determine that a knot is constructibly by isotopy.

Let $k$ be a doubly slice $(n-2)$-knot, $n \geq 3$, with double slice $D_{1}^{n-1}, D_{2}^{n-1}$, i.e., $k=\partial D_{i}^{n-1} \quad(i=1,2)$, where $D_{i}^{n-1} \subset B^{n+1}$ is an $(n-1)$-disk knot and $D_{1}^{n-1} \cup_{\partial} D_{2}^{n-1}$ is a trivial $(n-1)$-knot $K$ in $S^{n+1}=B^{n+1} \cup_{\partial} B^{n+1}$. Denote $\operatorname{cl}\left(S^{n}-\operatorname{nbd}(k)\right)$ and $\operatorname{cl}\left(B^{n+1}-\operatorname{nbd}\left(D_{i}^{n-1}\right)\right)$ by $X$ and $Y_{i}$, respectively. Notice that if $k$ is fibered, then any fibration of $k$ extends to a locally trivial fibration $f_{i}: \partial Y_{i} \rightarrow S^{1}$. (To see this, it is helpful to regard $\partial Y_{i}$ as the result of 0 -framed surgery on $k$.) If both $f_{1}$ and $f_{2}$ further extend to fibrations of $D_{1}^{n-1}$ and 
$D_{2}^{n-1}$, respectively, then we will call $D_{1}^{n-1}, D_{2}^{n-1}$ a fibered double slice for $k$. (Notice that $f_{1}$ and $f_{2}$ are only required to extend for some fibration of $k$.)

Theorem 2.1. Let $k$ be any doubly slice fibered $(n-2)-k n o t, n \geq 3$. If $k$ is constructible by isotopy, then $k$ has a fibered double slice. The converse is true, provided $n \geq 5$.

Proof. The first assertion is a consequence of the definition of construction by isotopy. The proof that we present is similar to arguments [A, pp. 61-62] and [Sil, p. 642].

After a small adjustment, if necessary, we can assume that the isotopy $h_{t}$ : $S^{n} \rightarrow S^{n}$ preserves not only the $(n-1)$-disk $\mathrm{D} \subset \partial \Xi$ but also a small $n$-ball $\mathbf{B} \subset S^{n}$ such that $\mathbf{B} \cap \Xi=\mathbf{D}$, with $h_{1}$ restricting to 1 on $\mathbb{B}$. Let $\mathbb{D}^{\prime}=\partial \mathbb{B}-$ int $\mathbb{D}$. We will see that $\mathbf{D}, \mathbf{D}^{\prime}$ is a fibered double slice for $k$.

Since $h$ is isotopic to 1 in the manner described above, there is an obvious diffeomorphism $\left(S^{n}-\right.$ int $\left.\mathbb{B}\right) \times_{h_{1}} S^{1} \rightarrow B^{n} \times S^{1}$ which maps $\left(\mathbb{D} \cup_{\partial} \mathbb{D}^{\prime}\right) \times \bar{\theta}$ to $S^{n-1} \times \bar{\theta}$. By suitably attaching a 2-handle $\left(\mathbb{D} \cup \cup_{\partial} \mathbb{D}^{\prime}\right) \times D^{2}$ to $\left(S^{n}-\operatorname{int} \mathbb{B}\right) \times{ }_{h_{1}} S^{1}$, we obtain $S^{n+1}$. We will identify the disk knots $\mathbb{D}, \mathbb{D}^{\prime}$ with $\mathbb{D} \times \overline{0}, \mathbb{D}^{\prime} \times \overline{0}$, respectively. Notice that $\mathbf{D} \cup \cup_{\partial} \mathbf{D}^{\prime}$ is a trivial $(n-1)$-knot in $S^{n+1}$.

Recall that $\mathbb{D}$ is a disk knot in the $(n+1)$-ball $\left(\Xi \times_{\phi} S^{1}\right) \cup \gamma$, where $\phi$ is the restriction of $h_{1}$ to $\Xi$ and $\gamma$ is a 2-handle $\left(=\mathbb{D} \times D^{2} \subset\left(\mathbb{D} \cup_{\partial} \mathbb{D}^{\prime}\right) \times D^{2}\right)$. Similarly, we can view $\mathbb{D}^{\prime}$ as a disk knot in the complementary $(n+1)$-ball $\left(\Xi^{\prime} \times{ }_{\phi^{\prime}} S^{1}\right) \cup \gamma^{\prime}$, where $\Xi^{\prime}=\operatorname{cl}\left(S^{n}-(\Xi \cup \mathbb{B})\right), \phi^{\prime}$ is the restriction of $h_{1}$ to $\Xi^{\prime}$ and $\gamma^{\prime}$ is the 2-handle $\mathbb{D}^{\prime} \times D^{2} \subset\left(\mathbb{D} \cup_{\partial} \mathbb{D}^{\prime}\right) \times D^{2}$. Then the fibration of $k$ clearly extends to fibrations of $\mathbb{D}$ and $\mathbb{D}^{\prime}$. Hence $\mathbb{D}, \mathbb{D}^{\prime}$ is a fibered double slice for $k$.

Conversely, suppose that $D_{1}^{n-1}, D_{2}^{n-1}$ is a fibered double slice for $k$. Let $F_{i} \quad(i=1,2)$ denote the respective fibers. Then $F_{1} \cap F_{2}$ is a fiber $F$ for the knot $k$. By combining the fibrations of $D_{1}^{n-1}$ and $D_{2}^{n-1}$, we can produce a fibration of the trivial $(n-1)$-knot $K=D_{1}^{n-1} \cup_{\partial} D_{2}^{n-1}$ with fiber $F_{1} \cup_{F}$ $F_{2}$. Since $K$ is trivial, $F_{1} \cup_{F} F_{2}$ must be an $n$-ball $B^{n}$. (Here we need the $h$-cobordism theorem, $n \geq 5$.) Also, the characteristic map $h: B^{n} \rightarrow$ $B^{n}$ must be pseudo-isotopic (rel $\partial$ ) to 1 ; i.e., there exists a diffeomorphism $H:\left(B^{n}, \partial B^{n}\right) \times I \rightarrow\left(B^{n}, \partial B^{n}\right) \times I$ such that $H(x, 1)=(h(x), 1), H(x, 0)=$ $(x, 0)$ and $H(x, t)=(x, t)$ for all $x \in \partial B^{n}$. By a relative version of Cerf's pseudo-isotopy theorem [Ce] there exists a 1-parameter family $H_{t}$ of pseudoisotopies (rel $\partial$ ) of $\left(B^{n}, \partial B^{n}\right)$ such that $H_{0}=1$ and $H_{1}=H$. Moreover, by elementary techniques (see [Mu, 5.16, 3.10, 3.9a]) we may assume that the flow $\mathrm{H}:\left(B^{n}, \partial B\right) \times I \times I \rightarrow\left(B^{n}, \partial B^{n}\right) \times I$, defined by $\mathrm{H}(x, s, t)=H_{t}(x, s)$ is smooth. Now the maps $H_{t}$ restricted to $\partial\left(B^{n} \times I\right)$ determine an isotopy of $S^{n}$, and using this it is clear that we obtain the knot $k$ with construction by isotopy. 
Remark. The above argument works also when $n=3,4$ provided we know that $F_{1} \cup_{F} F_{2}=B^{n}$ and $h$ is isotopic (rel $\partial$ ) to 1 .

In [Sil] we showed that every simple doubly slice fibered (2q-1)-knot, $q \geq 2$, and every simple $Z$-torsion-free doubly slice fibered $(2 q-2)$-knot, $q \geq 5$, is constructible by isotopy. The following corollary of Theorem 2.1 strengthens these results and shows that they depend only on fundamental group information.

Corollary 2.2. Let $k$ be any doubly slice fibered $(n-2)-k n o t, n \geq 5$. If $\pi_{1}\left(S^{n}-k\right) \cong Z$, then $k$ is constructible by isotopy.

Proof. Let $D_{1}^{n-1}, D_{2}^{n-1}$ be any double slice for $k$. By the van Kampen theorem $\tilde{X}_{i}(i=1,2)$ is simply connected. (Here $\sim$ denotes infinite cyclic cover.) Also, the Mayer-Vietoris sequence reveals that $H_{r}(\tilde{X}) \cong H_{r}\left(\tilde{X}_{1}\right) \oplus H_{r}\left(\tilde{X}_{2}\right), r>$ 0 . Since $k$ is fibered, $H_{*}(\tilde{X})$ is finitely generated over $Z$ in all dimensions. Hence so is $H_{*}\left(\tilde{X}_{i}\right)$. By Serre's theorem [Sp] $\pi_{*}\left(\tilde{X}_{i}\right)$ and hence $\pi_{*}\left(X_{i}\right)$ are finitely generated in all dimensions. Now by the fibration theorem of Browder and Levine [BrLe] the fibration of $\partial X_{i}$ extends to a fibration of $X_{i}$. Thus $D_{1}^{n-1}, D_{2}^{n-2}$ is a fibered double slice for $k$. By Theorem $2.1 k$ is constructible by isotopy.

The above proof of Corollary 2.2 shows that if $k$ is a doubly slice fibered $(n-2)$-knot, $n \geq 5$, and $\pi_{1}\left(S^{n}-k\right) \cong Z$, then any double slice for $k$ is, in fact, a fibered double slice. The next result demonstrates that if $\pi_{1}\left(S^{n}-k\right) \neq Z$, then this comfortable situation may not exist.

Proposition 2.3. For each $q \geq 3$ there exists a doubly slice fibered $(2 q-2)$ knot constructible by isotopy having infinitely many distinct invertible nonfibered slices. Moreover, the slices can be chosen to have the same fibered inverse slice.

Proof. Choose positive integers $c, m$ such that $m \neq 1,2,3,4,6, c^{q} \equiv 1$ $(\bmod m)$ and $c^{j}-1 \equiv$ unit $(\bmod m)$ if $0<j<q$. (An elementary argument such as that in [ $R_{2}$, proof of Corollary 2.4] shows that this can be done choosing $m$ to be the product of two appropriate distinct odd primes.) In [ $\mathbf{R}_{1}$ ] Ruberman constructs a fibered $(2 q-2)$-knot $k$ with fiber $F$ equal to the lens space $L\left(m ; 1, c, \ldots, c^{q-1}\right)$-minus-an-open- $(2 q-1)$-ball and characteristic map inducing multiplication by $c$ on $\pi_{1}(F) \cong Z_{m}$. Consider the doubly slice fibered knot $k=k_{1} \#-k_{1}$.

By [A] or Theorem 1.1 above, $k$ is constructible by isotopy. Recall that there exists an isotopy (rel $\mathbf{D}$ ) of $\Xi=F \times I$ in $S^{2 q}$ with final map $\phi$ such that $\left(\Xi \times{ }_{\phi} S^{1}\right) \cup \gamma=B^{2 q+1}$ and $\mathrm{D}(=\mathrm{D} \times \overline{0})$ is an invertible fibered slice for $k$. (Here $\mathbf{D}$ is a $(2 q-1)$-ball in $\partial \Xi$ and $\gamma$ is a 2-handle attached along $\mathbf{D} \times S^{1}$.) By the proof of Theorem 2.1 D has an inverse $D_{0}$ such that $D, D_{0}$ is a fibered double slice for $k$. We will modify the construction of $\mathbf{D} \subset B^{2 q+1}$ to produce the desired nonfibered slices for $k$. It will turn out that $\mathbf{D}_{0}$ is an inverse for those slices. 
By [Ba] the Whitehead group $\mathrm{Wh}\left(Z_{m}\right)$ is infinite free abelian and by [Mi] every element is self-conjugate. By [St] we can find a relative $h$-cobordism of $\Xi$ with any specified torsion $\tau \in \mathrm{Wh}\left(Z_{m}\right)$ and by composing two copies we can produce a relative $h$-cobordism $(V ; \Xi, \Xi)$ with torsion $\tau+(-1)^{2 q} \bar{\tau}=2 \tau$. (See [Mi, Lemma 11.4].) Consider the manifold $V /\{(x, 0) \sim(\phi(x), 1)\}$ obtained by identifying the two copies of $\Xi$ via $\phi$. The boundary of this new manifold is the same as $\partial\left(\Xi \times{ }_{\phi} S^{1}\right)$; in particular, it contains $\mathrm{D} \times S^{1}$ and so we can attach a 2-handle $\gamma$ as before. Call the result $W$. It is straightforward to check that $W \approx B^{2 q+1}$ and hence $\mathbf{D}(=\mathbf{D} \times \overline{0})$ is a slice $\mathbf{D}_{\tau}$ for the knot $k$.

To see that $\mathbf{D}_{\tau}$ has inverse $\mathbf{D}_{0}$, form the union $\left(W, \mathbb{D}_{\tau}\right) \cup_{\partial}\left(B^{2 q+1}, \mathbb{D}_{0}\right)$, identifying boundaries in the same manner as for $\left(B^{2 q+1}, \mathbb{D}\right) \cup_{\partial}\left(B^{2 q+1}, \mathbb{D}_{0}\right)$. The $(2 q-1)$-sphere $\mathbf{D}_{\tau} \cup_{\partial} \mathbf{D}_{0}$ still bounds a ball in $W \cup_{\partial} B^{2 q+1}$-in fact, the ball can be chosen to be the union of $\Xi$ and $\Xi^{\prime}$ as in the proof of Theorem 2.1. Unfortunately, $W \cup_{\partial} B^{2 q+1}$ may be a fake $(2 q+1)$-sphere $\Sigma$ instead of $S^{2 q+1}$; replacing the original $h$-cobordism $V$ by $V \#(-\Sigma)$, another $h$-cobordism with torsion $2 \tau$, eliminates the difficulty. Thus $\mathbb{D}_{0}$ is an inverse slice for $\mathbb{D}_{\tau}$.

Finally, we show that (1) $\mathbf{D}_{\tau}$ cannot be fibered unless $\tau=0$ and (2) $\mathbb{D}_{\sigma}, \mathbf{D}_{\tau}$ are distinct disk knots unless $\sigma=\tau$. These two assertions follow from Farrell's fibering theorem $[F]$, although here we shall use Siebenmann's refinement [Sie]: Let $G$ denote the fundamental group of

$$
\operatorname{cl}\left(W-\operatorname{nbd}\left(\mathbf{D}_{\tau}\right)\right) \approx V /\{(x, 0) \sim(\phi(x), 1)\}
$$

and consider the homomorphism $i_{*}: \mathrm{Wh}\left(Z_{m}\right) \rightarrow \mathrm{Wh}(G)$ induced by the quotient map $i: V \rightarrow \operatorname{cl}\left(W-\operatorname{nbd}\left(\mathbf{D}_{\tau}\right)\right)$. By [Sie, $\S 1$ and Lemma 4.4] $i_{*}(2 \tau)$ is a topological invariant of $\operatorname{cl}\left(W-\operatorname{nbd}\left(\mathbf{D}_{\tau}\right)\right)$ and a total obstruction to extending the fibration of the boundary to a fibration of $\operatorname{cl}\left(W-\operatorname{nbd}\left(\mathbb{D}_{\tau}\right)\right)$. Furthermore, by [Sie, $\S 8]$ or $[\mathrm{F}]$,

$$
\operatorname{ker} i_{*}=\text { image }\left[\mathrm{Wh}\left(Z_{m}\right) \stackrel{1-\phi_{*}}{\longrightarrow} \mathrm{Wh}\left(Z_{m}\right)\right] \text {. }
$$

Since $\phi$ induces multiplication by $c$ on $Z_{m}$, it follows from the definition of equivalence for elements of the Whitehead group that $\phi_{*}=1$. Hence $i_{*}$ is injective. Consequently, the slices $\mathbf{D}_{\tau}$ are distinct and cannot be fibered unless $\tau=0$.

\section{TWIST-SPINNING DOUBLY SLICE KNOTS}

In [Z] Zeeman introduced the technique of $m$-twist-spinning any $(n-2)$-knot $k$ in order to obtain a fibered $(n-1)$-knot $\tau^{m} k$. Since the $m$-twist-spin of any trivial knot is again trivial [ $\mathrm{Z}$, Corollary 3], it follows that the $m$-twist-spin of any doubly slice knot is a doubly slice fibered knot. In this section we show that any such knot is, in fact, constructible by isotopy.

It is convenient for our purpose to use Litherland's version [Li] of the twistspin construction. We will adopt the notation of [Li] wherever possible. 
Theorem 3.1. If $k$ is any doubly slice ( $n-2)-k n o t, n \geq 3$, then the m-twist-spun $k n o t \tau^{m} k$ is constructible by isotopy.

In [A] Aitchison asks whether construction by isotopy can yield a doubly slice knot that is not the double of any disk knot. (See Theorem 1.1.) We can now provide an affirmative answer:

Corollary 3.2. The 3-twist-spun knot $\tau^{3} 9_{46}$ is constructible by isotopy but is not the double of any disk knot.

Proof of Corollary 3.2. The 1-knot $9_{46}$ is doubly slice [HoT] and consequently $\tau^{3} 9_{46}$ is constructible by isotopy by Theorem 3.1. Levine [Le] has shown that $\tau^{3} 9_{46}$ is not the double of any 2-disk knot.

Remark. It is likely that other examples in higher dimensions can be produced using results of Ruberman [ $R_{2}$ ]. (See [Sm, remarks, p. 70].)

In order to prove Theorem 3.1 we need to describe the $m$-twist-spin $\tau^{m} D^{n-1}$ of any disk knot $D^{n-1} \subset B^{n+1}$. This extension of the twist-spin construction has been used previously $\left[R_{2}\right]$, but to our knowledge details of the construction have not appeared.

Let $D^{n-1} \subset B^{n+1}$ be any $(n-1)$-disk knot with boundary $k \subset S^{n}$. Let $D^{n-1} \times D^{2}$ be a tubular neighborhood of $D^{n-1}$ with exterior

$$
\mathbf{X}=\operatorname{cl}\left(B^{n+1}-\left(D^{n-1} \times D^{2}\right)\right),
$$

restricting to a tubular neighborhood $k \times D^{2}$ of $k$ with exterior $X$. (If $n=3$ we will always assume that $k \times x$ is null-homologous in $X$ for every $x \in \partial D^{2}$.) Let $\partial_{0} \mathbf{X} \times I$ be the collar neighborhood in $\mathbf{X}$ of $\partial_{0} \mathbf{X}=D^{n-1} \times \partial D^{2}$ such that $\left(\partial_{0} \mathbf{X} \times I\right) \cap X=\partial X \times I$. We will identify $\partial_{0} \mathbf{X}$ and $\partial X$ with $\partial_{0} \mathbf{X} \times 0$ and $\partial X \times 0$, respectively. Define a map $t:\left(B^{n+1}, D^{n-1}\right) \rightarrow\left(B^{n+1}, D^{n-1}\right)$ by $t(x \times \bar{\theta} \times \phi)=x \times(\overline{\theta+\phi}) \times \phi$ if $x \times \bar{\theta} \times \phi \in \partial_{0} \mathbf{X} \times I, t(y)=y$ if $y \notin \partial_{0} \mathbf{X} \times I$. Notice that $t$ restricts to a similarly defined map, also denoted $t$, on $\left(S^{n}, k\right)$.

Choose a small $(n-2)$-ball neighborhood $k_{-}$in $k$ of a base point $*$ and set $B_{-}^{n}=k_{-} \times D^{2}$. Then $\left(B_{-}^{n}, k_{-}\right)$is a standard ball pair. In [Li] the $m$-twist-spun knot $\tau^{m} k$ was defined using the complementary ball pair $\left(B_{+}^{n}, k_{+}\right)$:

$$
\left(S^{n+1}, \tau^{m} k\right)=\partial\left(B_{+}^{n}, k_{+}\right) \times B^{2} \cup_{\partial}\left(B_{+}^{n}, k_{+}\right) \times_{t^{m}} \partial B^{2} .
$$

Now extend $k_{-}$to an $(n-1)$-ball neighborhood $D_{-}^{n-1}$ of $*$ in $D^{n-1}$ such that $D_{-}^{n-1} \cap k=k_{-}$and set $B_{-}^{n+1}=D_{-}^{n-1} \times D^{2}$. Using the complementary ball pair $\left(B_{+}^{n+1}, D_{+}^{n-1}\right)$ we define $\tau^{m} D^{n-1}$ :

$$
\left(B^{n+2}, \tau^{m} D^{n-1}\right)=\left(\partial_{1} B^{n+1}, \partial_{1} D^{n-1}\right) \times B^{2} \cup_{\partial}\left(B_{+}^{n+1}, D_{+}^{n-1}\right) \times_{t^{m}} \partial B^{2} .
$$

Here $\partial_{1} D^{n-1}=\operatorname{cl}\left(\partial D_{+}^{n-1}-k_{+}\right)$and $\partial_{1} B^{n+1}=\operatorname{cl}\left(\partial B_{+}^{n+1}-B_{+}^{n}\right)$. One checks that $B^{n+2}$ and $\tau^{m} D^{n-1}$ are indeed balls. (See [Li, p. 321].) Moreover, the construction does not depend on the choice of base point or tubular neighborhood of $D^{n-1}$. 
It is not difficult to see that $\tau^{m} k$ has a tubular neighborhood $\partial k_{+} \times D^{2} \times B^{2} U$ $k_{+} \times D^{2} \times \partial B^{2}$ and that the exterior $X^{\prime}$ can be identified with $k_{-} \times \partial D^{2} \times B^{2} U$ $X \times_{t^{m}} \partial B^{2}$. In [Li] a specific fibration $X^{\prime} \rightarrow S^{1}$, trivial on $\partial X^{\prime}$, is described. Likewise, $\tau^{m} D^{n-1}$ has a tubular neighborhood $\partial_{1} D_{+}^{n-1} \times D^{2} \times B^{2} \cup D_{+}^{n-1} \times D^{2} \times$ $\partial B^{2}$ and the exterior $\mathbf{X}^{\prime}$ can be identified with $D_{-}^{n-1} \times \partial D^{2} \times B^{2} \cup \mathbf{X} \times{ }_{t^{m}} \partial B^{2}$. Examining the details of $[\mathrm{Li}, \S 4]$ it is readily seen that the fibration of $X^{\prime}$ extends to a fibration $\mathbf{X}^{\prime} \rightarrow S^{1}$, trivial on $\partial_{1} D_{+}^{n-1} \times \partial D^{2} \times B^{2} \cup D_{+}^{n-1} \times \partial D^{2} \times$ $\partial B^{2} \subset \partial \mathbf{X}^{\prime}$. (See also proof of Theorem 3.1 below.) Consequently, $\tau^{m} D^{n-1}$ is a fibered $n$-disk knot.

We are now ready to prove Theorem 3.1.

Proof of Theorem 3.1. Let $k$ be any doubly slice knot with double slice $D_{1}^{n-1}$, $D_{2}^{n-1}$; i.e., $D_{i}^{n-1} \subset B_{i}^{n+1}(i=1,2)$ is a disk knot with $\partial D_{1}^{n-1}=\partial D_{2}^{n-1}=k$ such that $U=D_{1}^{n-1} \cup_{\partial} D_{2}^{n-1}$ is a trivial $(n-1)$-knot in $S^{n+1}=B_{1}^{n+1} \cup_{\partial} B_{2}^{n+1}$. The trivial $m$-twist-spun knot $\tau^{m} U$ is the union of disk knots $\tau^{m} D_{1}^{n-1}, \tau^{m} D_{2}^{n-1}$ with intersection $\tau^{m} k$. Also, the fibration of $\tau^{m} k$ from the twist-spin construction extends to fibrations of $\tau^{m} D_{1}^{n-1}$ and $\tau^{m} D_{2}^{n-1}$. Hence $\tau^{m} D_{1}, \tau^{m} D_{2}$ is a fibered double slice for $\tau^{m} k$. By Theorem $2.1 \tau^{m} k$ is constructible by isotopy, provided $n \geq 4$.

In order to prove Theorem 3.1 when $n=3$, we must look more closely at the fibration of $\tau^{m} U$ and apply the remark following the proof of Theorem 2.1.

Choose a tubular neighborhood $U \times D^{2}$ for $U$ with exterior $\mathbf{Y}$. Assume that $U \times D^{2}$ restricts to tubular neighborhoods $D_{i}^{n-1} \times D^{2}$ (resp. $k \times D^{2}$ ) for $D_{i}^{n-1}$ (resp. $k$ ) with exteriors $\mathbf{X}_{i}$ (resp. $X$ ). Since $U$ is trivial, we may choose a fibration $p: \mathbf{Y} \rightarrow S^{1}$, trivial on $\partial \mathbf{Y}$, with characteristic map equal to 1 . Following [Li] and using the notation established above, we describe a specific fibration of $\tau^{m} U$.

The $m$-twist-spun knot $\tau^{m} U$ has tubular neighborhood $\partial U_{+} \times D^{2} \times B^{2} \cup U_{+} \times$ $D^{2} \times \partial B^{2}$ and its exterior $\mathbf{Y}^{\prime}$ can be identified with $U_{-} \times \partial D^{2} \times B^{2} \cup \mathbf{Y} \times{ }_{t^{m}} \partial B^{2}$. It is straightforward to see that $\mathbf{Y}^{\prime}$ is diffeomorphic to $U_{-} \times \partial D^{2} \times B^{2} \cup_{\alpha} \mathbf{Y} \times \partial B^{2}$, where $\alpha: x \times \bar{\theta} \times \bar{\phi} \rightarrow x \times(\overline{\theta+m \phi}) \times \bar{\phi}$; a specific diffeomorphism $\bar{H}$ is described in [Li]. Litherland shows that the map $q: \mathbf{Y} \times \partial B^{2} \rightarrow S^{1}$ defined by $q(y \times \phi)=p(y)-\overline{m \phi}$ is a fibration, and $q$ extends in an obvious way to a fibration $q: U_{-} \times \partial D^{2} \times B^{2} \cup_{\alpha} \mathbf{Y} \times \partial B^{2} \rightarrow S^{1}$. The induced map $\bar{q}=q \circ \bar{H}$ is now a fibration of $\tau^{m} U$. Notice that $\tau^{m} D_{i}^{n-1}(i=1,2)$ has a tubular neighborhood $\partial D_{i+}^{n-1} \times D^{2} \times B^{2} \cup D_{i+}^{n-1} \times D^{2} \times \partial B^{2}$ and its exterior $\mathbf{X}_{i}$ can be identified with $D_{i-}^{n-1} \times \partial D^{2} \times B^{2} \cup \mathbf{X}_{i} \times_{t^{m}} \partial B^{2}$. Under $\bar{H}$ the exterior $\mathbf{X}_{i}$ is diffeomorphic to $D_{i-}^{n-1} \times \partial D^{2} \times B^{2} \cup_{\alpha \mid} \mathbf{X}_{i} \times \partial B^{2}$, and clearly $q$ restricts to a fibration $q_{i}$. Now $\bar{q}_{i}=q_{i} \circ \bar{H}$ is a fibration of $\tau^{m} D_{i}^{n-1}$ and $\bar{q}_{1}, \bar{q}_{2}$ both restrict to the same fibration of the twist-spun knot $\tau^{m} k$. 
The fiber $q^{-1}(\overline{0})=U_{-} \times\{\overline{0}\} \times B^{2} \cup\left\{y \times \bar{\phi} \in \mathbf{Y} \times \partial B^{2} \mid p(y)=\overline{m \phi}\right\}$ is the union of fibers $q_{1}^{-1}(\overline{0}), q_{2}^{-1}(\overline{0})$, and the intersection $q_{1}^{-1}(\overline{0}) \cap q_{2}^{-1}(\overline{0})$ is a fiber of $\tau^{m} k$. As in [Li, Z], $q^{-1}(\overline{0})$ may be described as a punctured $m$-fold cyclic branched cover of $U$. Since $U$ is a trivial $(n-1)-\mathrm{knot}, q^{-1}(\overline{0})=B^{n}$. In $[\mathrm{Li}$, Z] it is shown that the characteristic map $h$ on $q^{-1}(\overline{0}) \cap \mathbf{Y} \times \partial B^{2}$ is given by $h(y \times \bar{\phi})=y \times\left(\overline{\phi+\frac{1}{m}}\right)$ and extended by rotation on each $x \times \overline{0} \times B^{2}$. Since $p$ was chosen to be a fibration of $U$ with trivial characteristic map, it is easy to see that $h$ is isotopic to 1 . (In $\mathbf{Y} \times \partial B^{2}$ one simply travels around the $\partial B^{2}$ factor, following the gradient vector-field of $p$.) In fact, the characteristic map $\bar{H}^{-1} \circ h \circ \bar{H}$ of the induced fibration $\bar{q}$ is isotopic rel $\partial$ to 1 .

Having now verified the requirements in the remark following the proof of Theorem 2.1, $\tau^{m} k$ is constructible by isotopy for all $n \geq 3$.

\section{A DOUBLY SLICE FIBERED KNOT THAT IS NOT CONSTRUCTIBLE BY ISOTOPY; A GEOMETRIC OBSTRUCTION}

We begin this final section with an observation: if an $(n-2)-$ knot $k$ is constructible by isotopy, then some capped-off fiber (i.e., fiber $\cup_{\partial}$ ball) of $k$ must embed in $S^{n}$. This is an immediate consequence of the construction, since $\partial \Xi \subset S^{n}$ is a capped-off fiber of $k$.

Unfortunately, above the classical dimension $n=3$ neither the fiber of an $(n-2)$-knot nor the capping-off process may be well defined. The fiber $F_{0}$ depends (up to a $s$-cobordism) on the choice of fibration, and the capped-off manifold $F=F_{0} \cup_{\partial} B^{n-1}$ depends (up to connected-sum with a homotopy sphere) on the choice of boundary identification. However, capping-off is well defined in the PL category. Consequently, we have the following obstruction to construction by isotopy. We shall see shortly that it is nontrivial:

Proposition 4.1. Let $k$ be any $(n-2)-k n o t, n \geq 7$. If $k$ is constructible by isotopy, then any capped-off fiber (corresponding to any fibration of $k$ ) embeds PL in $S^{n}$.

Proof. Denote $\operatorname{cl}\left(S^{n}-\operatorname{nbd}(k)\right)$ by $X$ and let $F_{0}=q^{-1}(\overline{0})$, where $q: X \rightarrow$ $S^{1}$ is the fibration from construction by isotopy. Then $F_{0}$, capped-off in an appropriate manner, is $\partial \Xi$ which embeds in $S^{n}$. Let $F_{0}^{\prime}=\left(q^{\prime}\right)^{-1}(\overline{0})$, where $q^{\prime}: X \rightarrow S^{1}$ is any fibration of $k$. We will show that $F^{\prime} \quad\left(=F_{0}^{\prime} \cup_{\partial} B^{n-1}\right)$ embeds PL in $S^{n}$.

Identify the infinite cyclic cover $\tilde{X}$ of $X$ with $F_{0} \times \mathbb{R}$. We can embed a copy of $F_{0}^{\prime}$ in $F_{0} \times[-N, N]$ by choosing $N$ sufficiently large. Notice that the $s$-cobordism $C$ between $\partial F_{0} \times\{-N\}$ and $\partial F_{0}^{\prime}$ is trivial, since $n \geq 7$. Attach $B^{n-1} \times[-N, N]$ to $\partial F_{0} \times[-N, N]$ in such a way that we see $F_{0}^{\prime} \subset \partial \Xi \times[-N, N]$. Since $\partial \Xi \subset S^{n}$, we can embed $\partial \Xi \times[-N, N] \subset S^{n}$ as a neighborhood of $\partial \Xi$. Now $F_{0}^{\prime} \cup C \cup B^{n-1} \times\{-N\} \subset S^{n}$ is PL homeomorphic to $F^{\prime}$. 
We are now ready to describe a doubly slice fibered 6-knot that is not constructible by isotopy. Let $L, L^{\prime}$ denote the 7-dimensional lens spaces $L\left(221 ; 1,21,(21)^{2},(21)^{3}\right), L(221 ; 1,21,4,4 \cdot 21)$, respectively. Ruberman $\left[\mathrm{R}_{1}\right]$ has shown that the punctured spaces $L_{0}=\operatorname{cl}\left(L-B^{7}\right), \quad L_{0}^{\prime}=\operatorname{cl}\left(L^{\prime}-B^{7}\right)$ embed in $S^{8}$ as fibers for $k=\partial L_{0}, k^{\prime}=\partial L_{0}^{\prime}$, respectively. In fact, the characteristic map for each of $k$ and $k^{\prime}$ induces the same homomorphism (multiplication by 21) on the fundamental group of the fiber. One can easily check (using [Co, p. 96], for example) that $L$ and $L^{\prime}$ are homotopy-equivalent. Hence by $\left[\mathrm{R}_{2}\right.$, Corollary 4.8$]$ the fibered $6-\mathrm{knot} k \#-k^{\prime}$ is doubly slice. One can also check (using [Co, p. 100]) that $L$ and $L^{\prime}$ are not diffeomorphic. By calculations of Cappell and Ruberman [CaR, p. 85], the capped-off fiber $L \#-L^{\prime}$ does not embed PL in $S^{8}$. Consequently, by Proposition 4.1, $k \#-k^{\prime}$ is not constructible by isotopy.

\section{QUESTIONS}

1. Is Aitchison's conjecture true for odd-dimensional knots; i.e., is every doubly slice fibered $(2 n-1)$-knot constructible by isotopy? This appears to be a difficult question for $n=1$.

2. If $k$ is any doubly slice fibered $(n-2)$-knot such that some capped-off fiber embeds in $S^{n}$, is $k$ constructible by isotopy? (Of course, if $n=3$ then any capped-off fiber embeds.)

3. If a $1-k n o t$ or $2-k n o t$ has a fibered double slice, is the knot constructible by isotopy?

4. Does the knot $k \#-k^{\prime}$ described in $\S 4$ have an invertible fibered slice?

\section{REFERENCES}

[A] I. R. Aitchison, Isotoping and twisting knots and ribbons, Ph.D. Dissertation, University of California at Berkeley, 1984.

[Ba] H. Bass, The stable structure of linear groups, Bull. Amer. Math. Soc. 70 (1964), 429-433.

[BrLe] W. Browder and J. Levine, Fibering manifolds over a circle, Comment. Math. Helv. 40 (1965), 153-160.

[CaR] S. Cappell and D. Ruberman, Imbeddings and homology cobordisms of lens spaces, Comment. Math. Helv. 63 (1988), 75-88.

[Ce] J. Cerf, La stratification naturelle des espaces de fonctions différentiables reeles et le théorème de la pseudo-isotopie, Inst. Hautes Études Sci. Publ. Math., no. 39.

[Co] M. Cohen, A course in simple-homotopy theory, Springer-Verlag, 1973.

[F] F. T. Farrell, The obstruction to fibering a manifold over a circle, Indiana Univ. Math. J. 21 (1971), 315-346.

[HiSil] L. R. Hitt and D. Silver, Families of ribbon knots via Stallings twists, Preprint.

[HoT] F. Hosokawa and H. Terasaka, On the unknotted sphere $S^{2}$ in $E^{4}$, Osaka J. Math. 13 (1961), 265-270.

[Le] J. Levine, Doubly slice knots and doubled disk knots, Michigan Math. J. 30 (1983), 249-256.

[Li] R. A. Litherland, Deforming twist-spun knots, Trans. Amer. Math. Soc. 250 (1979), 311-331.

[Mi] J. Milnor, Whitehead torsion, Bull. Amer. Math. Soc. 72 (1966), 358-426. 
[Mu] J. R. Munkres, Elementary differential topology, Ann. of Math. Stud., no. 54, Princeton Univ. Press, Princeton, N. J., 1966.

$\left[\mathrm{R}_{1}\right]$ D. Ruberman, Imbedding punctured lens spaces and connected sums, Pacific J. Math. 113 (1984), 481-491.

$\left[\mathbf{R}_{2}\right]$ _ The Casson-Gordon invariants in high-dimensional knot theory, Trans. Amer. Math. Soc. 306 (1988), 579-595.

[Sie] L. C. Siebenmann, A total Whitehead obstruction to fibering over a circle, Comment. Math. Helv. 45 (1970), 1-48.

[Sil] D. Silver, On Aitchison's construction by isotopy, Trans. Amer. Math. Soc. 305 (1988), 641652.

[Sm] L. Smolinsky, Double disk knots and a link invariant, Ph. D. Dissertation, Brandeis Univ., Waltham, Mass., 1985.

[Sp] E. H. Spanier, Algebraic topology, McGraw-Hill, 1966.

[St] J. Stallings, On infinite processes leading to differentiability in the complement of a point, Differential and Combinatorial Topology (A Symposium in honor of M. Morse), Princeton Univ. Press, Princeton, N. J., 1965, pp. 245-254.

[Z] E. C. Zeeman, Twisting spun knots, Trans. Amer. Math. Soc. 115 (1965), 471-495.

Department of Mathematics and Statistics, University of South Alabama, Mobile, Alabama 36688 